Ann. Biol. anim. Bioch. Biophys., 1978, 18 (5), 1117-1128.

\title{
Effets comparés, à très court terme, des acides (n-9) cis et trans docosénoïques sur les lipides cardiaques du rat : influence de l'apport en acide linoléique
}

\author{
par P. O. ASTORG, Geneviève COMPOINT
}

Station de Recherches sur la Qualité des Aliments de l'Homme, I.N.R.A., 7 rue Sully, 21034 Dijon Cedex.

Summary. Cardiac lipids : Very short-ferm effects of dietary $(n-9)$ cis and trans docosenoic acids in rat as related to varying amounts of linoleic acid.

Male weanling rats were fed semi-synthetic diets for 4 days. These diets contained 15 p. 100 lipids, of which 7.5 p. 100 was tribrassidin (trans-C22: 1) or trierucin (cis-C22:1) with amounts of linoleic acid (C18:2) varying between 0 and 3 p. 100. Heart contents in triglycerides (TG) and phospholipids (PL) and the fatty acid composition of these were determined. In contrast to erucic acid, brassidic acid induced no great triglyceride accumulation in the heart, and incorporated into heart lipids to a much lesser extent than its cis isomer. These differences were not due to the lower intestinal absorption of the trans isomer. The highest dietary levels of $\mathrm{C} 18: 2$ tended to increase either the cardiac TG content (with erucic acid) or the incorporation of C22 : 1 into the TG (with brassidic acid). Trans fatty acids, absent in the diet, were found in heart lipids of brassidic acid-fed rats (C16:1, $\mathrm{C} 20: 1$ and mainly $\mathrm{C} 18: 1)$, thus indicating that brassidic acid, like erucic acid, was partly metabolized to shorter monoenes.

\section{Introduction.}

De nombreux travaux mettent en évidence la stéatose cardiaque produite, à très court terme, par l'acide érucique (cis, n-9 docosénoïque) chez le Rat. Plusieurs auteurs (Astorg et al., 1974 ; Astorg et Cluzan, 1975 ; Beare-Rogers et al., 1971 ; Beare-Rogers et Nera, $1972 a, b)$ ont observé que les huiles de colza ou de poisson partiellement hydrogénées, riches en isomères de l'acide érucique, notamment en isomères trans, ont, de ce point de vue, un effet moins marqué que l'huile de colza non hydrogénée. Un travail récent de Rocquelin ef al. (1975) indique que l'acide brassidique, isomère trans de l'acide érucique, est moins stéatogène que l'acide érucique, et montre qu'il s'incorpore beaucoup moins que ce dernier, à très courtterme, dans les lipides du cœur.

Par ailleurs, dans un de nos travaux antérieurs sur les huiles de poisson hydrogénées (Astorg ef al. ,1974 ; Astorg et Cluzan, 1975), il est apparu qu'une supplémentation en acide linoléique a un effet inhibiteur sur la séatose cardiaque induite par ces huiles ef sur l'incorporation de l'acide doco sénoïque dans les lipides de l'organe. 
Le but du présent travail est d'une part, de confirmer le résultat de Rocquelin ef al. concernant le faible effet stéatogène de l'acide brassidique et, d'autre part, d'essayer de préciser l'influence du niveau d'apport en acide linoléique sur l'intensité de la lipidose produite par l'acide érucique ou l'acide brassidique.

\section{Matériel et méthodes.}

Expérimentation sur animaux. Cinquante-cinq rats mâles Wistar, EOPS, pris au sevrage, sont placés en cages individuelles ef nourris pendant 3 jours avec une provende commerciale. On les répartit alors en 11 lots de 5 , qui reçoivent pendant 4 jours des régimes semi-synthétiques équilibrés $\left.{ }^{(}{ }^{1}\right)$, ne différant que par la nature des corps gras qu'ils contiennent (15 p. 100 en poids). Ces corps gras sont : 1 ) l'huile d'arachide (lot témoin) ; 2) cinq mélanges de tribrassidine, de trioléine et d'huile de maïs, dans des proportions telles que la teneur des mélanges en acide brassidique soił de 50 p. 100 environ, et leurs teneurs en acide linoléique atteignent respectivement $0,2,4,10$ et 20 p. 100 environ (lots $B_{0}, B_{2}, B_{4}, B_{10}$ et $B_{20}$ ) ; 3) cinq mélanges analogues, la triérucine remplaçant la tribrassidine (lots $E_{0}, E_{2}, E_{4}, E_{10}$ et $E_{20}$ ). La composition en acides gras de ces mélanges est indiquée dans le tableau 1.

\section{TABLEAU 1}

Composition en acides gras des lipides des régimes (p. 100 en poids des esters méthyliques)

\begin{tabular}{|c|c|c|c|c|c|c|c|c|c|}
\hline & & $C 16: 0$ & C18:0 & $C 18: 1$ & C18: 2 & $C 20: 1$ & $C 22: 0$ & $\begin{array}{c}\mathrm{C} 22: 1 \\
\mathrm{Cis}\end{array}$ & $\begin{array}{c}\text { C22: } 1 \\
\text { trans }\end{array}$ \\
\hline Huile d'arachide & A.... & 9,5 & 3,7 & 57,3 & 21,6 & 1,6 & 2,6 & 0,2 & - \\
\hline Tribrassidine (B) & 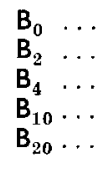 & $\begin{array}{l}0,6 \\
0,5 \\
0,8 \\
1,3 \\
1,9\end{array}$ & $\begin{array}{l}1,3 \\
1,5 \\
1,3 \\
1,4 \\
1,8\end{array}$ & $\begin{array}{l}47,8 \\
44,1 \\
42,9 \\
35,5 \\
22,5\end{array}$ & $\begin{array}{r}\overline{1,5} \\
4,2 \\
10,3 \\
18,4\end{array}$ & $\begin{array}{l}- \\
- \\
-\end{array}$ & $\begin{array}{l}0,6 \\
0,9 \\
0,8 \\
0,8 \\
1,3\end{array}$ & $\begin{array}{l}- \\
- \\
-\end{array}$ & $\begin{array}{l}49,7 \\
51,6 \\
49,9 \\
50,5 \\
53,8\end{array}$ \\
\hline Triérucine (E) & $\begin{array}{ll}E_{0} & \cdots \\
E_{2} & \cdots \\
E_{4} & \cdots \\
E_{10} & \cdots \\
E_{20} & \cdots\end{array}$ & $\begin{array}{l}0,5 \\
0,6 \\
0,9 \\
1,4 \\
2,2\end{array}$ & $\begin{array}{l}1,3 \\
1,2 \\
1,5 \\
1,6 \\
1,9\end{array}$ & $\begin{array}{l}47,2 \\
46,0 \\
43,6 \\
37,4 \\
26,0\end{array}$ & $\begin{array}{r}- \\
2,2 \\
4,4 \\
10,8 \\
22,2\end{array}$ & $\begin{array}{l}1,2 \\
1,0 \\
1,0 \\
1,0 \\
1,0\end{array}$ & $\begin{array}{l}0,4 \\
0,4 \\
0,5 \\
0,6 \\
0,5\end{array}$ & $\begin{array}{l}48,7 \\
47,3 \\
47,2 \\
46,6 \\
45,3\end{array}$ & $\begin{array}{l}- \\
- \\
-\end{array}$ \\
\hline
\end{tabular}

Les consommations de nourriture sont mesurées individuellement sur tous les animaux. Les fèces sont collectées, pendant la durée du régime expérimental, chez les rats des lots $B_{0}, B_{20}, E_{0}$ et $E_{20}$. En fin d'expérience, les rats sont sacrifiés par décapitation, après 5 à 6 heures de jeûne. Les cœurs sont prélevés, pesés et słockés à $-18^{\circ} \mathrm{C}$ dans le chloroforme/méthanol $2 / 1 \mathrm{~V} / \mathrm{V}$, en vue d'analyses sur les lipides.

(1) Pour plus de détails sur la composition du régime, cf. Astorg et Cluzan, 1976. 
Analyse des lipides ef des acides gras du cœur et des fèces $\left(^{(1)}\right.$. - Les lipides de chaque cœur sont extraits et purifiés selon Folch ef al. (1957). Sur un pool de 5 extraits par lot, on isole les triglycérides (TG) et les phospholipides (PL) par chromatographie semipréparative sur couche mince de gel de silice (solvant : hexane/éther éthylique/ méthanol/acide acétique $90 / 20 / 1 / 2, V / V)$. La composition en acides gras de ces classes lipidiques, ainsi que celle des lipides totaux, est déterminée par chromatographie gaz-liquide (CGL) des esters méthyliques correspondants sur colonnes conventionnelles garnies de phases stationnaires polaires (BDS, DEGS). Pour les échantillons issus des rats ayant ingéré du C22 : 1 trans, des analyses supplémentaires sont effectuées sur colonnes capillaires garnies d'Apiezon $L$ (inox, $50 \mathrm{~m} \times 0,25 \mathrm{~mm}, 200$ à $240^{\circ} \mathrm{C}$ ) ou sur colonnes conventionnelles garnies de SP 2340 [inox, $6 \mathrm{~m} \times 2,5 \mathrm{~mm}$, 15 p. 100 SP 2340 sur chromosorb P 100/120 mesh (fabrication Supelco), $225^{\circ} \mathrm{C}$ ] ; ces deux types de colonnes permettent de séparer de façon satisfaisante les isomères géométriques des acides gras monoinsaturés. Le dosage des acides gras et des classes (TG, PL) est réalisé par la méthode du standard interne (dans le cas des classes séparées sur couches minces, on utilise deux standards : pentadécanoate et heptadécanoate de méthyle).

Les lipides fécaux sont extraits par le mélange chloroforme/méthanol/acide chlorhydrique $12 \mathrm{~N} \mathrm{100/50/5} \mathrm{V} / \mathrm{V}$, saponifiés, et, après extraction de l'insaponifiable, les acides gras sont dosés par pesée. Leur composition est déterminée par CGL selon les techniques citées plus haut.

Analyse statistique des résultats. - D'une façon générale, les grandeurs mesurées ont fait l'objet d'une analyse de variance à deux critères de classification, correspondant aux deux facteurs étudiés : forme géométrique du C22 : 1 (acide brassidique/ acide érucique), teneur du régime en acide linoléique. Dans le cas des grandeurs concernant les classes lipidiques (triglycérides et phospholipides) pour lesquelles on ne dispose que d'une valeur par lot, on s'est contenté d'une analyse de variance sans étude de l'interaction, analogue à l'analyse de variance « en blocs »; dans certains cas, la présence d'une interaction plus ou moins nette limite la portée de cette méthode. Pour l'éłude des effets des facteurs étudiés sur le CUD du C22:1, on a effectué une analyse de covariance excrété/ingéré.

Dans les tableaux de résultats, on indique, pour chaque grandeur étudiée, l'écart-type commun, puis les valeurs de $F$ correspondant aux facteurs étudiés (acide brassidique/acide érucique, noté $B / E$; teneur du régime en acide linoléique, noté $\mathrm{C} 18$ : 2) et, le cas échéant, à leur interaction, ainsi que leur degré de signification. Les calculs ont été réalisés avec une calculatrice Olivetti P602, à l'aide de programmes mis au point par Lowy et Manchon.

\section{Résultats.}

Quantités de C22: 1 absorbées (tabl. 2).

Ces déterminations ont été faites sur 4 des 11 lots : $B_{0}, B_{20}, E_{0}$ et $E_{20}$. Le niveau de consommation de nourriture est plus élevé dans le cas de régimes contenant de la

(1) Pour plus de détails sur ces techniques, cf. Astorg et Cluzan, 1977. 
tribrassidine (lots $B_{0}$ et $B_{20}$ ), ce qui compense partiellement la médiocre utilisation digestive de l'acide brassidique lorsqu'il est ingéré sous cette forme. Les quantités de C22 : 1 absorbées demeurent cependant plus élevées pour l'acide érucique que pour l'acide brassidique, surtout chez le lot $\mathrm{E}_{0}$. On note par ailleurs une diminution du coefficient d'utilisation digestive apparent (CUD) ( ${ }^{1}$ ) de l'acide érucique lorsqu'on remplace, dans le mélange avec la triérucine, la trioléine $\left(E_{0}\right)$ par de l'huile de maïs $\left(E_{20}\right)$ (les CUD calculés ne peuvent être comparés qu'entre eux ; ils n'ont que peu de signification dans l'absolu, les conditions expérimentales étant trop éloignées de celles requises pour la détermination d'un CUD, notamment en ce qui concerne la durée de l'essai).

TABLEAU 2

Quantités de C22 : 1 ingérées, excrétées ef absorbées ( $\mathrm{mg}$ d'esters méthyliques/4 jours) ; CUD du C22 : 1 (moyennes sur 5 rats)

\begin{tabular}{|c|c|c|c|c|c|c|c|}
\hline & \multicolumn{4}{|c|}{ Lots expérimentaux } & \multicolumn{3}{|c|}{ Analyse de variance } \\
\hline & $\mathrm{B}_{0}$ & $\mathrm{~B}_{20}$ & $E_{0}$ & $E_{20}$ & $B / E$ & $C 18: 2$ & $\begin{array}{l}\text { Inter- } \\
\text { action }\end{array}$ \\
\hline Ingéré & $3552 \pm 131$ & $3798 \pm 161$ & $3032 \pm 120$ & $2714 \pm 99$ & $19,10 * *$ & 0,04 & 2,36 \\
\hline Excrété & $1478 \pm 120$ & $1573 \pm 128$ & $152 \pm 14$ & $268 \pm 54$ & n. d. & n. d. & n. $d$ \\
\hline Absorbé & $2074 \pm 74$ & $2225 \pm 113$ & $2880 \pm 107$ & $2446 \pm 146$ & $20,65 * *$ & 1,57 & $6,65 *$ \\
\hline CUD (1) & $58,6 \pm 2,39$ & $58,6 \pm 2,52$ & $95,0 \pm 0,27$ & $89,9 \pm 3,16$ & $55,50 * *$ & 0,22 & $5,56 *$ \\
\hline
\end{tabular}

* Significatif pour $P \leqslant 0,05$; ** Significatif pour $P \leqslant 0,01 ; n$. d. = non déterminé.

(1) On a effectué une analyse de covariance excrété/ingéré (la régression est significative). En raison de l'hétérogénéité des variances des excrétés, la significativité de l'interaction $(F=5,56)$ ne nous semble pas assurée.

Teneurs du cœur en lipides totaux, triglycérides, phospholipides, exprimés par leur contenu en acides gras (tabl. 3).

L'acide brassidique est loin de produire l'accumulation massive de triglycérides (10 fois la valeur témoin) observée chez les animaux ayant ingéré de l'acide érucique. Il entraîne cependant, par rapport aux valeurs obtenues pour le lot témoin (arachide), une augmentation sensible (non testable statistiquement) de la teneur du cœur en triglycérides, répercutée au niveau de la teneur en acides gras totaux (effet non significatif) ; ces variations sont cependant peu marquées. La différence d'effet des deux isomères du $\mathrm{C} 22$ : 1 est bien trop importante pour être impułable à la différence des quantités absorbées (cf. plus haut). Par ailleurs, dans le cas de l'acide érucique (lots $\mathrm{E}$ ), les apports élevés en acide linoléique dans le régime (lots $E_{10}$ et surtout $E_{20}$ ) ont tendance à renforcer l'action stéatogène de l'acide érucique (effet non significatif),

(1) CUD $=\frac{\text { ingéré-excrété }}{\text { ingéré }} \times 100$ 
en dépit du fait que la quantité de $C 22: 1$ absorbée soit moins élevée dans le lot $E_{20}$ que dans le lot $E_{0}$. La teneur du cœur en phospholipides ne varie pas en fonction du régime.

\section{TABLEAU 3}

Teneurs du cour ( $m g$ d'esters méthyliques/g de cœeur) en acides gras fotaux (moyennes sur 5 rats), acides gros des triglycérides ef acides gras des phospholipides (pools de 5 rats)

\begin{tabular}{|c|c|c|c|c|c|c|c|c|}
\hline & & \multicolumn{2}{|c|}{ Acides gras totaux } & \multicolumn{2}{|c|}{$\begin{array}{c}\text { Acides gras } \\
\text { des triglycérides }\end{array}$} & \multicolumn{3}{|c|}{$\begin{array}{c}\text { Acides gras } \\
\text { des phospholipides }\end{array}$} \\
\hline Huile d'arachide & A...... & $20,8 \pm 0,21$ & & 3,5 & & 15,4 & & \\
\hline Tribrassidine (B) & $\left\{\begin{array}{lll}\mathrm{B}_{0} & \ldots \\
\mathrm{B}_{2} & \ldots \ldots \\
\mathrm{B}_{4} & \ldots \\
\mathrm{B}_{4} & \ldots \\
\mathrm{B}_{10} & \ldots \\
\mathrm{B}_{20} & \ldots\end{array}\right.$ & $\left.\begin{array}{l}22,1 \pm 2,55 \\
25,7 \pm 3,20 \\
24,0 \pm 0,90 \\
25,3 \pm 3,12 \\
26,3 \pm 1,10\end{array}\right\}$ & $24,7 \pm 1,07$ & $\left.\begin{array}{r}6,8 \\
11,0 \\
8,1 \\
8,4 \\
7,7\end{array}\right\}$ & $8,4 \pm 0,70$ & $\left.\begin{array}{l}15,2 \\
16,7 \\
16,4 \\
15,4 \\
15,7\end{array}\right\}$ & 15,9 & $9 \pm 0,29$ \\
\hline Triérucine (E) & $\begin{cases}E_{0} & \ldots \\
E_{2} & \cdots \\
E_{4} & \cdots \\
E_{10} & \cdots \\
E_{20} & \cdots\end{cases}$ & $\left.\begin{array}{l}52,0 \pm 2,88 \\
52,3 \pm 5,80 \\
48,7 \pm 3,74 \\
56,4 \pm 4,36 \\
67,1 \pm 5,52\end{array}\right\}$ & $55,3 \pm 2,05$ & $\left.\begin{array}{l}32,7 \\
30,8 \\
28,8 \\
37,2 \\
44,6\end{array}\right\}$ & $34,8 \pm 2,81$ & $\left.\begin{array}{l}14,9 \\
14,1 \\
13,0 \\
17,5 \\
17,7\end{array}\right\}$ & 15,4 & $4 \pm 0,93$ \\
\hline Ecart-type commu & un ..... & 3,66 & 1,64 & 4,58 & 2,05 & & 54 & 0,69 \\
\hline \multicolumn{9}{|c|}{ Analyse de variance } \\
\hline $\begin{array}{c}\text { (lot } A \text { exclus) } \\
(1)\end{array}$ & $\begin{array}{l}\mathrm{E} \ldots \ldots \\
18: 2 \ldots \\
\text { teraction }\end{array}$ & \multicolumn{2}{|c|}{$\begin{array}{c}174,90 * * \\
2,56 \\
1,44\end{array}$} & \multicolumn{2}{|c|}{$\begin{array}{l}72,59 * * \\
0,75 \\
-\end{array}$} & \multicolumn{3}{|c|}{$\begin{array}{l}0,15 \\
0,47 \\
-\end{array}$} \\
\hline
\end{tabular}

** Significatif pour $p \leqslant 0,01$.

(1) L'analyse de variance à un critère de classification sur les teneurs en acides gras totaux (ou leurs logarithmes), lot $A$ inclus, ne permet pas de mettre en évidence une différence significative entre l'ensemble des lots $B$ et le lot $A$.

Principaux acides gras des lipides tofaux et des triglycérides du cœur (tabl. 4 et 5).

a) La stéatose cardiaque provoquée par l'acide érucique (lots $\mathrm{E}$ ) correspond essentiellement à une accumulation d'acide érucique et d'acide oléique, principaux acides gras des triglycérides, et secondairement d'acide linoléique, qui s'incorpore notablement à cette classe lorsqu'il est abondant dans le régime (lot $E_{\mathbf{2 0}}$ ). L'acide brassidique (lots B) s'incorpore beaucoup moins dans les triglycérides cardiaques que son isomère cis : 6-15 p. 100 contre 42-46 p. 100 ( tabl. 5). Sa teneur dans le cœur est 15 à 20 fois plus faible que celle de l'acide érucique (tabl. 4) ; par gramme d'absorbé, l'incorporation de ce dernier est plus de 10 fois plus élevées que celle de l'acide brassidique [C22: 1 incorporé ( $\mathrm{mg} / \mathrm{g}$ de cœur) /C22: 1 absorbé $\left(\mathrm{g} / 4\right.$ jours) : $\mathrm{B}_{0}: 0,56$, $\left.B_{20}: 0,89, E_{0}: 6,50, E_{20}: 10,60\right]$.

b) La teneur du régime en acide linoléique semble influer sur l'incorporation du C22 : 1 dans le cœur, aussi bien avec l'acide érucique qu'avec l'acide brassidique. 


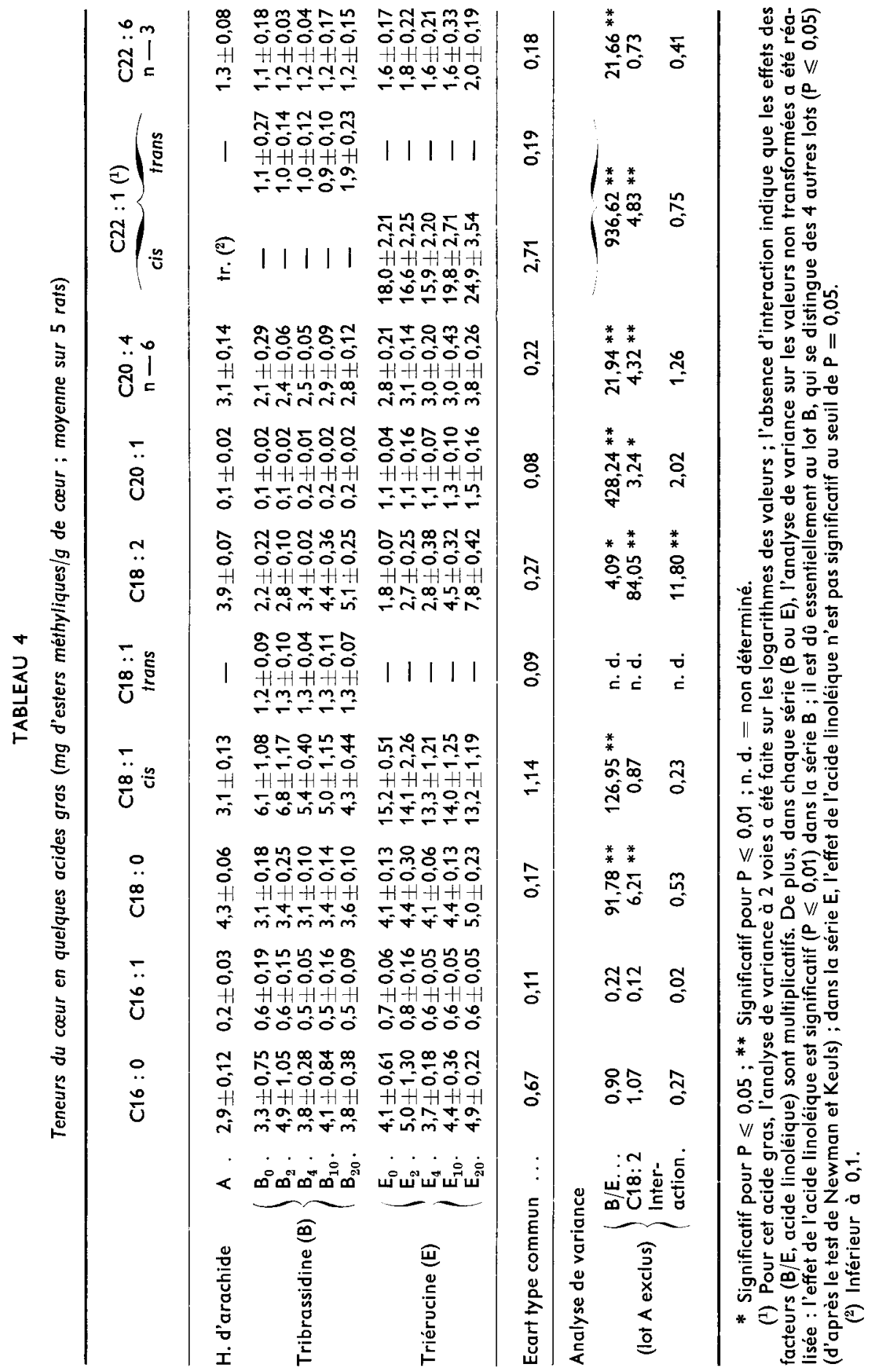


Dans les deux cas, cet effet est surtout dû au niveau d'apport le plus élevé en acide linoléique, qui provoque une augmentation de la teneur du cœur en $C 22: 1$, mais selon les modalités différentes suivant le cas : avec l'acide brassidique, on observe une augmentation de la concentration du $C 22: 1$ dans les triglycérides (de 6-8 à 14 p. 100, tabl. 5), la teneur du cœur en ces derniers demeurant constante; avec l'acide érucique, inversement, la teneur du cœur en TG augmente, le pourcentage de C22:1 dans les acides gras de cette classe variant peu.

c) Des acides gras monoinsaturés trans (autres que le $C 22: 1$ ), totalement absents des régimes, apparaissent chez les rats des lots B : C16:1 (à l'état de traces), $\mathrm{C} 20: 1$ (en petites quantités), mais surtout C18: 1 (acide élaïdique) ; l'incorporation de ce dernier ( 5 p. 100 des acides gras totaux, 3-4 p. 100 des triglycérides) s'effectue uniquement aux dépens de l'acide stéarique. Les triglycérides ne contiennent qu'environ 1/4 de l'acide élaïdique présent dans le cœur, le reste (3/4) étant incorporé aux phospholipides (voir plus loin).

d) L'acide arachidonique (C20:4n-6) et l'acide docosahexaénoïque (C22:6 $n-3)$ semblent un peu plus abondants dans les lipides cardiaques des lots $E$ que dans ceux des lots B (effet significatif). Par ailleurs, la teneur du cœur en C20:4 augmente sensiblement avec l'apport alimentaire en acide linoléique.

Acides gras des phospholipides du cœur (tabl. 6). - Les résultats sont donnés en composition centésimale, la teneur du cœur en phospholipides (PL) étant à peu près la même dans tous les lots.

a) L'acide palmitique est un peu plus abondant dans les $\mathrm{PL}$ de la série $B$ que dans ceux de la série $E(+1$ à 2 p. 100).

b) Chez les rats de la série $B$, on trouve 6 à 7 p. 100 d'acide élaïdique dans les acides gras des PL. Par rapport aux lots de la série $E$ (et lot témoin A), la proportion d'acide stéarique est abaissée de la même valeur. L'apportalimentaire en acide linoléique est sans influence sur le phénomène. Du $C 16: 1$ trans et du $C 20: 1$ trans apparaissent également, en petites quantités, dans les $\mathrm{PL}$ des lots $\mathrm{B}$.

c) Lorsque le taux d'acide linoléique dans le régime s'accroît, la teneur des PL en acide oléique diminue, et leur teneur en $C 18: 2$ augmente. Ces deux variations, d'une amplitude de 10 p. 100 lorsque l'apport alimentaire en $C 18: 2$ passe de 0 à 20 p. 100 des lipides, se neutralisent l'une l'autre, aussi bien dans la série B que dans la série $\mathrm{E}$ (C18:1 cis + $\mathrm{C} 18: 2 \simeq$ constante). La teneur des PL en acide oléique est sensiblement la même dans les deux séries $\left(^{1}\right)$, alors que le $C 18: 2$ est toujours nettement plus abondant chez les rats des lots B que chez ceux des lots $E$ ( +4 à 6 p. 100).

d) L'incorporation du C22 : 1 dans les $P L$, indépendante de la richesse du régime en $\mathrm{C} 18: 2$, est nettement plus élevée pour l'isomère cis que pour l'isomère trans (même en tenant compte de la différence d'absorbé) : 5-6 p. 100 contre 2 p. 100. Cette différence compense quantitativement la différence en sens inverse observée pour le $\mathrm{C} 18: 2$ (cf. c).

e) On ne retrouve pas ici les effets constatés sur les teneurs du cœur en C20:4 et en $\mathrm{C} 22$ : 6 (cf. tabl. 4) ; ces effets peuvent être dus à d'autres classes lipidiques, non étudiées ici.

(') La différence constatée en faveur des lots $E$ est très faible, bien que significative (łabl. 6). 


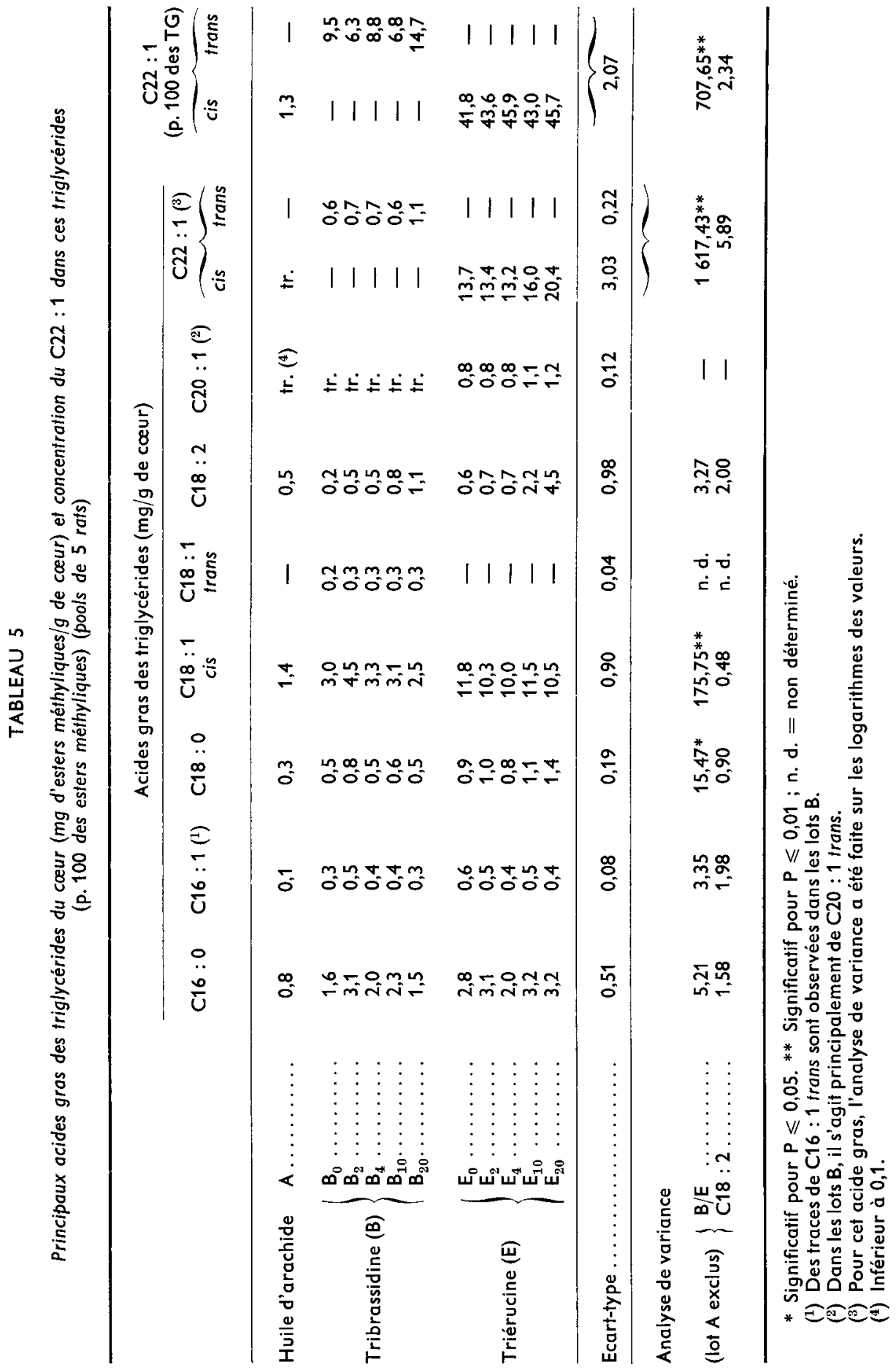




\section{Discussion.}

L'acide brassidique, au contraire de son isomère cis, n'entraîne pas d'accumulation massive de triglycérides dans le myocarde du Rat, et s'incorpore beaucoup moins dans les lipides de l'organe : nous confirmons ici pleinement les résultats de Rocquelin et al. (1975). La forme géométrique de la double liaison des acides docosénoïques paraît donc déterminante dans cet aspect de leurs effets physiologiques, alors que, selon d'autres auteurs, la position de cette double liaison importe moins, tout au moins en ce qui concerne les acides gras cis (Beare-Rogers et Nera 1972 ; Craig et BeareRogers, 1967, Vles et al., 1974). Mattson et Streck (1974) n'observent pas de stéatose cardiaque chez des rats ayant ingéré de l'acide behénique (C22:0) pendant 7 jours. A cette réserve près que l'expérience en question n'est pas décisive, en raison du faible taux d'absorption du C22:0, on est tenté de mettre en parallèle la similitude de structure des acides béhénique et brassidique (molécule rectiligne ou à peu près rectiligne) et l'analogie de leurs effets sur le coeur : la structure cis docosénoĩque (courbure de la molécule au niveau de la double liaison) pourrait être déterminante dans l'induction de la stéatose.

L'effet d'un apport élevé en acide linoléique, qui entraîne soit une augmentation de la teneur du cœeur en triglycérides (cas de l'acide érucique), soit une incorporation accrue du C22 : 1 dans les triglycérides (cas de l'acide brassidique), va à l'encontre d'un résultat précédent (Astorg et al., 1974 ; Astorg et Cluzan, 1975), où une addition d'huile de maïs à une huile de hareng hydrogénée entraînait une diminution de la lipidose ef de l'incorporation du C22 : 1 dans les lipides du cœur. Aucune explication satisfaisante ne peut actuellement être fournie sur cette apparente contradiction. II apparaît seulement que le niveau de l'apport en acide linoléique n'est pas indifférent : d'autres travaux seront nécessaires pour clarifier ce point.

Comme Rocquelin et al. (1975), nous constatons que l'acide brassidique est en partie transformé en monoènes plus courts (C20:1, C18:1, C16:1), puisqu'on trouve ces derniers dans les lipides du cœur. Cette transformation, bien démontrée en ce qui concerne l'acide érucique (Boucrot et Bézard, 1973 ; Carreau et al., 1968), a été également constatée avec d'autres acides gras en C22 : 1 (Conacher et al., 1973 ; Craig et Beare-Rogers, 1967). II s'agirait donc d'un processus assez général de la métabolisation des acides docosénoïques, assuré nołamment par les systèmes enzymatiques microsomaux du foie (Boucrot et Bezard, 1973 ; Carreau et al., 1968). C'est l'acide élaïdique qui se trouve le plus abondamment incorporé dans les lipides du cœur, notamment dans les phospholipides : cette observation est en accord avec des travaux antérieurs (Le Breton et Lemarchal, 1967), montrant l'affinité de cet acide gras pour la position 1 des phospholipides, où il entre en compétition avec l'acide stéarique.

Enfin, dans un de nos travaux récents (Astorg et Levillain, 1977), il apparaît que l'acide brassidique ne possède pas les propriétés cardiopathogènes à moyen terme de son isomère cis, Cette différence dans les effets sur le myocarde, tant à court qu'à moyen terme, pourrait être due à une meilleure utilisation de l'acide brassidique par cet organe que celle, faible, de l'acide érucique (Cheng et Pande, 1975 ; Christophersen et Bremer, 1972 ; Houtsmuller et al., 1970 ; Lemarchal ef al., 1972). Les premiers 


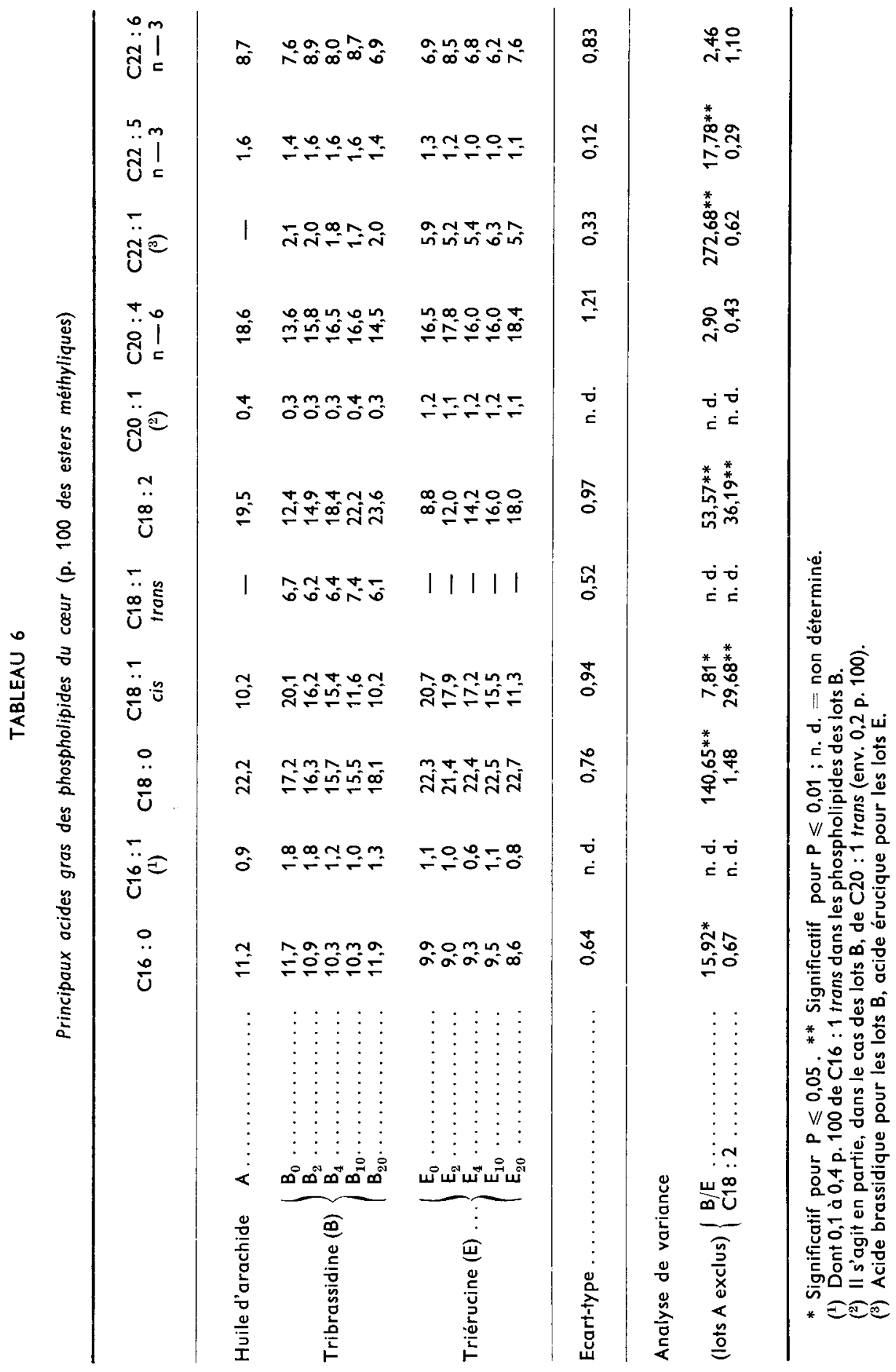


travaux sur ce point, cependant, ne confirment pas cette interprétation (Christiansen et al., 1977 ; Korsrud et al., 1977), encore que l'acide brassidique inhibe moins que son isomère cis la $\beta$-oxydation de l'acide palmitique par la mitochondrie cardiaque (Christiansen ef al., 1977). Une autre hypothèse, n'excluant pas la première, serait que la métabolisation de l'acide brassidique en monoènes plus courts est plus rapide que celle de l'acide érucique.

Reçu en décembre 1977. Accepté en mars 1978.

Remerciements. - Cette étude a été réalisée avec l'aide financière de l'INSERM (Action thématique programmée no 13-74-34). Les corps gras ont été fournis par la société Astra-Calvé.

\section{Références}

ASTORG P. O., CLUZAN R., 1975. Effets comparés, à très court terme, d'huiles riches en acide docosénoïque sur les lipides cardiaques du rat : huile de colza et huiles de hareng partiellement hydrogénées. C. R. Soc. Biol. Fr., 169, 966-970.

ASTORG P. O., CLUZAN R., 1976. Effets comparés d'huiles de hareng partiellement hydrogénées et de l'huile de colza chez le rat. I. Effets à moyen terme sur la croissance, le poids des organes ef l'histologie du cœur. Ann. Nutrit. Aliment., 30, 581-602.

ASTORG P. O., CLUZAN R., 1977. Effets physiologiques comparés d'huiles de hareng partiellement hydrogénées et de l'huile de colza chez le rat. II. Effets à très court terme et à moyen terme sur les lipides du cœur, du foie et du tissu adipeux. Ann. Nutrit. Aliment., 31, 43-68.

ASTORG P. O., LEVILLAIN R., 1977. Lésions du myocarde après ingestion d'acide docosénoïque chez le rat : effets comparés des acides brassidique et érucique. C. R. Acad. Sci. Paris, sér. D, 285, 1123-1126.

ASTORG P. O., ROCQUELIN G., CAUSERET J., 1974. Valeur nutritionnelle et effets physiopathologiques d'huiles de hareng partiellement hydrogénées, 97-109. In Effets physiopathologiques des acides gras à chaine très longue, Coll. de synthèse, sér. Action thématique $n^{\circ} 2$, INSERM, Paris.

BEARE-ROGERS J. L., NERA E. A., 1972a. Accumulation of cardiac fatty acids in rats fed synthetized oils containing C22 fatty acids. Lipids, 7, 46-50.

BEARE-ROGERS J. L., NERA E. A., 1972b. Cardiac lipids in rats and gerbils fed oils containing C22 fatty acids. Lipids, 7, 548-552.

BEARE-ROGERS J. L., NERA E. A., HEGGTVEIT H. A., 1971. Cardiac lipid changes in rats fed oils containing long-chain fatty acids. Can. Inst. Food Technol. J., 4, 120-124.

BOUCROT P., BEZARD J., 1973. Incorporation et transformations métaboliques de l'acide érucique${ }^{14} \mathrm{C}$, in vivo, dans les lipides de plusieurs organes et tissus de rats soumis à un régime à base d'huile de colza, ou de canbra, ou d'arachide. Arch. Sci. physiol., 27, 1-24.

CARREAU J. P., THORON A., LAPOUS D., RAULIN J., 1968. Métabolisme de l'acide érucique. I. Conversion en acide oléique. Bull. Soc. Chim. biol., 50, 1973-1981.

CHENG C. K., PANDE S. V., 1975. Erucic acid metabolism by rat heart preparations. Lipids, 10, 335-339.

CHRISTIANSEN R. Z., CHRISTOPHERSEN B. O., BREMER J., 1977. Monoethylenic C20 and C22 fatty acids in marine oil and rapeseed oil. Studies on their oxydation and on their relative ability to inhibit palmitate oxidation in heart and liver mitochondria. Bioch. biophys. Acta, 487, $28-36$.

CHRISTOPHERSEN B. O., BREMER J., 1972. Erucic acid : and inhibitor of fatty acid oxydation in the heart. Bioch. biophys. Acta, 280, 506-514.

CONACHER H. B. S., PAGE B. D., BEARE ROGERS J. L., 1973. Monoethylenic fatty acid isomers in cardiac lipids of rats fed partially hydrogenated herring oil. Lipids, 8, 256-258.

CRAIG B. M., BEARE-ROGERS J. L., 1967. The $\beta$-oxydative degradation of docosenoïc acids to eicosenoïc and octadecenoīc acids in the rat. Can. J. Biochem. Physiol., 45, 1075-1079. 
FOLCH J., LEES M., SLOANE-STANLEY G. H., 1957. A simple method for the isolation and purification of total lipids from animal tissues. J. biol. Chem., 226, 497-509.

HOUTSMULLER U. M. T., STRUIJK C. B., VAN DER BEEK A., 1970. Decrease in rate of ATP synthe sis of isolated rat heart mitochondria induced by dietary erucic acid. Biochim. biophys. Acta, 218, 564-566.

KORSRUD G. O., CONACHER H. B. S., JARVIS G. A., BEARE-ROGERS J. L., 1977. Studies on long chain cis and trans acyl-COA esters and acyl-COA dehydrogenase from rat heart mitochondria. Lipids, 12, 177-181.

LE BRETON E., LEMARCHAL P., 1967. Les acides gras de forme trans en physiologie animale. Nouvelles acquisitions. Coh. Nutr. Diét., 2, 41-48.

LEMARCHAL P., CLOUET P., BLOND J. P., 1972. $\beta$-oxydations comparées des acides érucique ef oléĭque par les mitochondries de cœur de rat in vitro. C. R. Acad. Sci. Paris, sér. D, 274, 1961 1963.

LOWY R., MANCHON P. Programmes d'analyses statistiques pour calculatrice Olivetti Programma 602 (non publiés).

MATTSON F. H., STRECK J. A., 1974. Effect of the consumption of glycerides containing behenic acid on the lipid content of the heart of weanling rats. J. Nutr., 104, 483-488.

ROCQUELIN G., JUANEDA P., PELERAN J. C., ASTORG P. O., 1975. Effets comparés à très court terme, des acides $\mathrm{n}-9$, trans-docosénoïques (brassidique) et $\mathrm{n}-9$, cis-docosénoïque (érucique) sur les lipides cardiaques du rat sevré. Nutr. Métabol., 19, 113-126.

VLES R. O., HOUTSMULLER U. M. T., TEN HOOR F., 1974. Recherches récentes sur les effets physiopathologiques des huiles de crucifères, 337-354. In Effets physiopathologiques des acides gras à chaîne très longue, Coll. de synthèse, sér. Action thématique $n^{\circ} 2$, INSERM, Paris. 